\title{
On certain inequalities for the prime counting function
}

\author{
József Sándor \\ Department of Mathematics, Babeș-Bolyai University \\ Cluj-Napoca, Romania \\ e-mail: jsandor@math.ubbcluj.ro
}

Received: 19 March 2021

Accepted: 23 October 2021

Abstract: We study certain inequalities for the prime counting function $\pi(x)$. Particularly, a new proof and a refinement of an inequality from [1] is provided.

Keywords: Prime counting function, Inequalities, Hardy-Littlewood conjecture.

2020 Mathematics Subject Classification: 11A25, 11A41.

\section{Introduction}

Let $\pi(x)$ denote the number of primes $\leq x$, where $x \geq 1$ is an integer. The famous HardyLittlewood conjecture states that the inequality

$$
\pi(x+y) \leq \pi(x)+\pi(y)
$$

is valid for all $x, y \geq 2$. Neither a proof nor a counterexample is known up to now.

There exist many inequalities in the literature, related to (1). For a survey of results, see the recent paper [1] of the author and H. Alzer and M. K. Kwong.

Many earlier results on $\pi(x)$ can be found in Chapter VII of the monograph [4]. For connections with other arithmetic functions, see the recent book [5] (see pp. 159-160).

One of the main results, proved in [1], is the inequality (see Theorem 1 of [1])

$$
\pi^{2}(x+y) \geq \frac{16}{9} \pi(x) \cdot \pi(y)
$$

where $x, y \geq 2$ and with equality only for $x=y=5$. 
For $x=y$ in (2), we get the result that

$$
\pi(2 x) \geq \frac{4}{3} \cdot \pi(x)
$$

with equality only for $x=5$. This is a converse of the famous Landau inequality

$$
\pi(2 x) \leq 2 \pi(x), \quad(x \geq 2) .
$$

Another result of [1] is the following (see left-hand side of Theorem 6 of [1]):

$$
\frac{1}{2} \leq \frac{\pi(x)^{x /(x+y)} \cdot \pi(y)^{y /(x+y)}}{\pi(x+y)} .
$$

The aim of this paper is to prove that, a converse inequality of (1) holds true, and this gives a new proof, as well as a refinement of (2). Another result will be motivated by relation (5).

\section{Main results}

The following classical inequality due to Rosser and Schoenfeld [3] will be used:

Lemma For all $x \geq 67$ one has

$$
\frac{x}{\log x-\frac{1}{2}}<\pi(x)<\frac{x}{\log x-\frac{3}{2}}
$$

The first main result of this paper gives a converse to inequality (1):

Theorem 1. For all $x, y \geq 2$ one has

$$
\pi(x+y) \geq \frac{2}{3} \cdot[\pi(x)+\pi(y)]
$$

with equality only for $(x, y)=(5,5) ;(3,7) ;(7,3)$.

Proof. Let $f(x)=\frac{x}{\log x-\frac{3}{2}}$. We shall prove that, this function is strictly concave for $x>e^{\frac{7}{2}}$. Indeed, one has $f^{\prime}(x)=\left(\log x-\frac{5}{2}\right) /\left(\log x-\frac{3}{2}\right)^{2}$, and after some elementary computations, we get $f^{\prime \prime}(x) . x .\left(\log x-\frac{3}{2}\right)^{3}=-\log x+\frac{7}{2}<0$ if $\log x>\frac{7}{2}$, i.e., $x>e^{\frac{7}{2}} \approx 33.11 \ldots$.

The concavity of $f(x)$ gives the inequality:

$$
f(x)+f(y) \leq 2 f\left(\frac{x+y}{2}\right) \text { for all } x, y \geq e^{\frac{7}{2}} .
$$

By the right-hand side of (6) and (8) we can write:

$$
\pi(x)+\pi(y)<f(x)+f(y) \leq \frac{x+y}{\log \left(\frac{x+y}{2}\right)-\frac{3}{2}} .
$$

Now, by the left-hand side of (6) one has $\frac{3}{2} \pi(x+y)>\frac{3}{2} \cdot \frac{x+y}{\log (x+y)-\frac{1}{2}}$, so at a first step, in attempt to have (7), we want to prove the inequality:

$$
\frac{x+y}{\log \left(\frac{x+y}{2}\right)-\frac{3}{5}}<\frac{3}{2} \cdot \frac{x+y}{\log (x+y)-\frac{1}{2}},
$$


which is equivalent with

$$
\log (x+y)>3 \log 2+\frac{9}{5}-\frac{1}{2}=3.379 \ldots,
$$

i.e., $x+y>e^{3.379 \ldots} \approx 29.3 \ldots$.

This is clearly true, if $x, y \geq 67$. Therefore, inequality (7) is proved for all $x, y \geq 67$.

Now, suppose that $x \geq y$ and $y \leq 66$. Then $\pi(y) \leq 18$, so $\frac{2}{3} \cdot[\pi(x)+\pi(y)] \leq \frac{2}{3} \cdot[\pi(x)+18]=$ $\frac{2}{3} \pi(x)+12$. We have to prove that $\frac{2}{3} \pi(x)+12 \leq \pi(x+y)$, or

$$
2 \pi(x)+36 \leq 3 \pi(x+y) .
$$

As $3 \pi(x) \leq 3 \pi(x+y)$, it will be sufficient to consider the inequality $2 \pi(x)+36 \leq 3 \pi(x)$, i.e., $\pi(x) \geq 36$. This is true, if $x \geq 151$.

Finally, we have to verify the case:

$$
2 \leq y \leq x \leq 150, y \leq 66
$$

This can be verified by a computer (for example, a Maple 13 program). This finishes the proof of Theorem 1.

\section{Corollary 1.}

$$
\pi^{2}(x+y) \geq \frac{4}{9} \cdot[\pi(x)+\pi(y)]^{2} \geq \frac{16}{9} \pi(x) \cdot \pi(y),
$$

which is a refinement of inequality (2).

Remark 1. For $y \leq x$ there is equality in the first inequality of (13) for $y=3, x=7$ and $y=5, x=5$; while in the second inequality only for $y=5, x=5$.

Indeed, the first inequality follows by (7), while the second one by $(a+b)^{2} \geq 4 a . b$, where $a=\pi(x), b=\pi(y)$.

Now, by the weighted arithmetic mean—geometric mean inequality one has:

$$
u^{\alpha} \cdot v^{\beta} \leq \alpha \cdot u+\beta . v
$$

for $u, v, \alpha, \beta>0 ; \alpha+\beta=1$. By letting $u=\pi(x), \alpha=x /(x+y), v=\pi(y), \beta=y /(x+y)$, by (5) and (14) we get

$$
\pi(x+y) \leq 2 . \pi(x)^{x /(x+y)} \cdot \pi(y)^{y /(x+y)} \leq 2\left[\frac{x}{x+y} \pi(x)+\frac{y}{x+y} \pi(y)\right],
$$

i.e.,

$$
(x+y) \cdot \pi(x+y) \leq 2 \cdot[x \cdot \pi(x)+y \cdot \pi(y)] .
$$

In 2001, Panaitopol [2] proved the inequality:

$$
\pi^{2}(x+y) \leq 2 \cdot\left[\pi^{2}(x)+\pi^{2}(y)\right]
$$

Motivated by these two inequalities, in what follows, we shall prove: 
Theorem 2. For all $x, y \geq 2$ one has

$$
\pi^{2}(x+y) \leq \frac{8}{7} \cdot[x \cdot \pi(x)+y \cdot \pi(y)]
$$

with equality only for $(x, y)=(3,4) ;(4,3)$.

Proof. Let us consider the function $g(x)=\frac{x^{2}}{\log x-\frac{1}{2}}(x>0)$. After elementary computations we can deduce that

$$
\frac{1}{2} \cdot g^{\prime \prime}(x) \cdot\left(\log x-\frac{1}{2}\right)^{2}=\log ^{2} x-\frac{3}{2} \log x+1
$$

Letting $\log x=t$, clearly $t^{2}-\frac{3}{2} t+1>0$ (having a negative discriminant), so we get that the function $g(x)$ is strictly convex.

By the left-hand side of (6) one has

$$
x \pi(x)+y \pi(y)>g(x)+g(y) \geq 2 g\left(\frac{x+y}{2}\right)=\left(\frac{x+y}{2}\right)^{2} /\left(\log \left(\frac{x+y}{2}\right)-\frac{1}{2}\right),
$$

by the convexity of $g(x)$.

By the right-hand side of (6), in order to prove (17), we have first to consider the validity of inequality

$$
\frac{8}{7} \cdot \frac{(x+y)^{2}}{4 \cdot\left[\log (x+y)-\log 2-\frac{1}{2}\right]}>\frac{(x+y)^{2}}{\left(\log (x+y)-\frac{3}{2}\right)^{2}} .
$$

Letting $\log (x+y)=m$, this becomes after elementary computations:

$$
2 m^{2}-13 m+7 \log ^{2}+8>0 .
$$

Solving this quadraatic inequality, it follows that it is true for $m>2.64 \ldots$, i.e., $x+y>e^{2.64 \ldots}=$ $14.01 \ldots$, which is clearly true for $x, y \geq 67$.

Now, let $x \geq y$ and $y \leq 66$. As $y \pi(y) \geq 2$, it is sufficient to consider the inequality:

$$
(\pi(x)+18)^{2} \leq \frac{8}{7} \cdot[x \pi(x)+2]
$$

This can written as $7 \pi^{2}(x)+252 \pi(x)+2268 \leq 8 x \pi(x)+16$. Now $8 x \pi(x) \geq 12 \pi^{2}(x)$ by the elementary inequality

$$
\frac{\pi(x)}{x} \leq \frac{2}{3} x, \quad(x \geq 2)
$$

Therefore, we have to consider

$$
5 \pi^{2}(x)-252 \pi(x)-2252 \geq 0
$$

which is valid for $\pi(x) \geq 38$, i.e., $x \geq 163$. 
It remains to verify inequality (17) for

$$
2 \leq y \leq x \leq 163
$$

This can be verified by a computer, but we can could reduce the numbers of verifications as follows:

Segal [6] proved in 1962 that inequality (1) holds true for any $x, y \geq 2$ and $x+y \leq 101081$.

Thus we can write for the values from (22) that

$$
7 \pi^{2}(x+y) \leq 7 \pi^{2}(x)+7 \pi^{2}(y)+14 \pi(x) \cdot \pi(y) .
$$

Now, if we can prove that $8 x \pi(x) \geq 14 \pi^{2}(x)$, then we would have $8 x \pi(x)+8 y \pi(y) \geq$ $14 \pi^{2}(x)+14 \pi(y)$ and inequality (23) would follow on base of $7 a^{2}+7 b^{2}>14 a b$ (i.e., $7(a-b)^{2}>$ $0)$ for $a=\pi(x), b=\pi(y)$.

The inequality $8 x \pi(x) \geq 14 \pi^{2}(x)$ is in fact

$$
\pi(x) \leq \frac{4}{7} x
$$

which is similar to (21), and is valid for all $x \geq 6$.

This is a simple exercise, so (22) can be reduced to

$$
2 \leq y \leq x \leq 5
$$

For these cases, even a verification by hand can be done. This finishes the proof of (17).

Remark 2. The constants $2 / 3$ and $8 / 7$ in Theorems 1 and 2 are the best possible. In a forthcoming paper some other inequalities of a new type will be presented.

\section{References}

[1] Alzer, H., Kwong, M. K., \& Sándor, J. (2021). Inequalities for $\pi(x)$. Rendiconti del Seminario Matematico della Università di Padova, 145(2), 1-15 (to appear).

[2] Panaitopol, L. (2001). Some generalizations for a theorem by Landau. Mathematical Inequalities \& Applications, 4(3), 327-330.

[3] Rosser, J. B., \& Schoenfeld, L. (1962). Approximate formulas for some functions of prime numbers. Illinois Journal of Mathematics, 6(1), 64-94.

[4] Sándor, J., Mitrinovic, D. S., \& Crstici, B. (2006). Handbook of Number Theory. I, Springer.

[5] Sándor, J., \& Atanassov, K. T. (2021). Arithmetic Functions, Nova Science Publishers, New York.

[6] Segal, S. L. (1962). On $\pi(x+y) \leq \pi(x)+\pi(y)$. Transactions of the American Mathematical Society, 104, 523-527. 